\title{
Dendrosomal Curcumin Suppresses Metastatic Breast Cancer in Mice by Changing M1/M2 Macrophage Balance in the Tumor Microenvironment
}

\author{
Sadaf Shiri'1,2, Ali Mohammad Alizadeh ${ }^{2 *}$, Behzad Baradaran ${ }^{1 *}$, Baharak \\ Farhanghi $^{2}$, Dariush Shanehbandi ${ }^{1}$, Saeed Khodayari ${ }^{2}$, Hamid Khodayari ${ }^{2}$, \\ Abbas Tavassoli ${ }^{3}$
}

\begin{abstract}
Curcumin, a lipid-soluble compound extracted from the plant Curcuma Longa, has been found to exert immunomodulatory effects via macrophages. However, most studies focus on the low bioavailability issue of curcumin by nano and microparticles, and thus the role of macrophages in the anticancer mechanism of curcumin has received little attention so far. We have previously shown the potential biocompatibility, biodegradability and anti-cancer effects of dendrosomal curcumin (DNC). In this study, twenty-seven BALB/c mice were equally divided into control as well as 40 and $80 \mathrm{mg} / \mathrm{kg}$ groups of DNC to investigate the involvement of macrophages in the antitumor effects of curcumin in a typical animal model of metastatic breast cancer. At the end of intervention, the tumor volume and weight were significantly reduced in DNC groups compared to control $(P<0.05)$. Histopathological data showed the presence of macrophages in tumor and spleen tissues. Real-time PCR results showed that DNC increased the expression of STAT4 and IL-12 genes in tumor and spleen tissues in comparison with control $(P<0.05)$, referring to the high levels of M1 macrophages. Furthermore treatment with DNC decreased STAT3, IL-10 and arginase I gene expression $(P<0.05)$, indicating low levels of M2 macrophage. The results confirm the role of macrophages in the protective effects of dendrosomal curcumin against metastatic breast cancer in mice.
\end{abstract}

Keywords: Dendrosomal curcumin - macrophage - breast cancer - BALB/c mice

Asian Pac J Cancer Prev, 16 (9), 3917-3922

\section{Introduction}

The heterogeneity of macrophages empowers them to efficiently toggle their phenotype and function to be able to respond to environmental cues. There are two major macrophage subsets known as M1 and M2 (Murray and Wynn, 2011; Biswas et al., 2012). Classical or M1 macrophages are characterized by high capacity to present antigens and secretion of high levels of IL-12 but low levels of IL-10 (Hao et al., 2012). IL-12 is one of the principal anti-tumor cytokines (Xu et al., 2010) that induces tyrosine phosphorylation of Janus Kinase family. This leads to the activation of signal transducer and activator of transcription 4 (STAT4) (Colombo and Trinchieri, 2002), which has been demonstrated to have a crucial role in anti-tumor immune responses (Schindler et al., 2001). Contrary to the M1 subset, M2 macrophages are incapable of presenting antigens and eliciting adaptive immune responses, and are described by low levels of IL-12 (Solinas et al., 2009; Hao et al., 2012) and high levels of IL-10 resulting in the phosphorylation of signal transducer and activator of transcription 3 (STAT3) (Sica et al., 2006). STAT3 upregulation has been reported in a variety of solid tumor such as breast cancer (Yu and Jove, 2004; Kamran et al., 2013), where it contributes to tumor progression (Niu et al., 2005). Therefore, M2 is known as tumor promoter and is also considered to be involved in promotion of tissue remodeling (Sinha et al., 2007; Tu et al., 2012; Cui et al., 2013; Alizadeh et al., 2014). In this regard, tumor associated macrophages (TAMs) exhibit several properties of M2 macrophages, and the ratio of IL12 and IL10 production is important to distinguish M1 and M2 (Martinez et al., 2007). Additionally, expression of arginase I is a key marker of M2 macrophage (Olefsky and Glass, 2010). A number of studies have already demonstrated that drugs and natural compounds including curcumin can have a positive effect on the immune system via macrophages.

Curcumin is a lipid-soluble compound extracted from plant Curcuma Longa and has been shown in several studies, to prevent cancer development with yet no discernible toxicity (Kelloff et al., 2000; Aggarwal et al., 2007). However, while several studies have designated

${ }^{I}$ Immunology Research Center, ${ }^{2}$ Cancer Research Center, Tehran University of Medical Sciences, ${ }^{3}$ Department of Pathology, Faculty of Veterinary Medicine, Tehran University, Tehran,Iran*For correspondence: aalizadeh@razi.tums.ac.ir, baradaranb@tbzmed.ac.ir 
Sadaf Shiri et al

curcumin as an immunosuppressive agent (Jagetia and Aggarwal, 2007; Varalakshmi et al., 2008; Aggarwal and Sung, 2009; Bhattacharyya et al., 2010), some others have reported its immunopotentiating effects (Antony et al., 1999; Jagetia and Aggarwal, 2007; Aggarwal and Sung, 2009) through macrophages. In recent years, many studies have focused on the pharmacokinetics and low bioavailability of curcumin in systemic circulation. Subsequently, many methods have been tested to increase the bioavailability, for instance using DNC (Alizadeh et al., 2012; Mohsenikia et al., 2013; Ghalandarlaki et al., 2014). An important advantage is that the structure of DNC monomers can be altered to optimize the formulation with regards to the biocompatibility and interaction features (Sadeghizadeh et al., 2008). Our previous results have shown the biocompatibility, biodegradability and anticancer effects of DNC in biological systems (Sarbolouki et al., 2000; Alizadeh et al., 2012; Babaei et al., 2012; Khaniki et al., 2013). In this study, we aimed to understand the mechanism of action of curcumin, particularly its differential effects on M1 and M2 macrophages in a typical animal model of metastatic breast cancer.

\section{Materials and Methods}

\section{Materials}

Curcumin was purchased from Merck Company (Darmstadt, Germany) with a purity of $95 \%$. The polymeric nanocarrier was locally produced in our lab (Patent Number: 71753). Phosphate-buffered saline (PBS) solution, Ketamine and Xylazine were purchased from Sigma Aldrich Co. (St Louis, MO, USA). Dulbecco's modified Eagle's Medium (DMEM), fetal bovine serum (FBS), penicillin and streptomycin were from Invitrogen.

\section{Dendrosomal curcumin preparation}

For DNC preparation, we used the optimized protocol in our lab as previously described (Tahmasebi et al., 2013). Briefly, different weight/weight ratios of DNC ranging from 50:1 to 10:1 were examined before settling a suitable ratio of 7:1. Curcumin was dissolved in various amounts of dendrosomes and checked for absorbance spectra by UV spectrophotometery (TECAN, Switzerland). Then, the appropriate mixture of dendrosomes and curcumin were evaluated for excitation/emission value in comparison with curcumin dissolved in PBS and 1\% methanol as control samples. The loading of dendrosomal nanocarriers with curcumin molecules was performed using a protocol from Gou et al. (2011). Briefly, curcumin and dendrosome were co-dissolved in $5 \mathrm{ml}$ of acetone; this solution was added into $5 \mathrm{ml}$ of PBS while stirring constantly. Then, acetone was evaporated in rotary evaporator. The dendrosome/ curcumin micelle solution was sterilized using a $0.22 \mu \mathrm{m}$ syringe filter (Millex-LG, Millipore Co. USA). Finally, prepared DNC was stored in $4^{\circ} \mathrm{C}$ in a light protected condition until use. For in vitro experiments, DNC was diluted in complete culture medium as mentioned for each assay.

\section{Cell lines and culture condition}

Metastatic breast cancer cell line (4T1) was obtained from the national cell bank of the Pasteur Institute, Tehran, Iran. The cells were cultured in Gibco ${ }^{\circledR}$ high glucose DMEM, and were supplemented with 10\% FBS, $100 \mathrm{U} /$ $\mathrm{mL}$ penicillin, and $100 \mu \mathrm{g} / \mathrm{mL}$ streptomycin . All cells were grown at $37^{\circ} \mathrm{C}$ in a humidified atmosphere with $5 \% \mathrm{CO}_{2}$.

\section{Animals}

All the animal studies have been conducted according to relevant national and international guidelines of the Weatherall report, and Institutional Animal Care and Use Committee (IACUC) of Tehran University of Medical Sciences. Animals were housed in pens exceeding the stipulated size requirements. All inbred female BALB/c mice (6-8 weeks old, purchased from Iran Pasteur Institute) were maintained in large group houses under 12-h dark and light cycles and were given access to food and water ad libitum.

\section{Tumorgenicity}

4T1 cells were trypsinized and re-suspended in 10-fold excess culture medium. After centrifugation, cells were re-suspended in PBS, and the cells were injected $\left(1 \times 10^{6}\right.$, $0.1 \mathrm{ml}$, s.c) using a 21-gauge needle in the left flank of BALB/c mice under Ketamine and Xylazine $(10 \mathrm{mg} / \mathrm{kg}$, i.p) anesthesia. Two-day after cell injection, twenty-seven mice were equally divided into three groups of 40 and 80 $\mathrm{mg} / \mathrm{kg}$ doses of DNC, and control group (injected with saline). DNC was administered for 35 consecutive days after tumor injection from day 3 up to day 38. All animals were euthanatized at day 42 post cell injection. Tumor volume was weekly measured by a digital vernier caliper (Mitutoyo, Japan) and calculated by using the following formula (Mohsenikia et al., 2013).

$\mathrm{V}=1 / 6$ ( $\pi$ LWD), where $\mathrm{L}=$ length, $\mathrm{W}=$ width, and $\mathrm{D}=$ depth.

\section{Histological assay}

In order to observe macrophages, the internal organs comprising the spleen and breast tumor were fixed in $10 \%$ formaldehyde, passaged and embedded in paraffin. Paraffin blocks were then sectioned by 3-5 $\mu \mathrm{m}$ thickness for hematoxylin and eosin ( $\mathrm{H} \& \mathrm{E}$ ) staining (Alizadeh et al., 2012). Slides were studied under OLYMPUS-BX51 microscope.

RNA extraction and real-time polymerase chain reaction

Total RNAs in spleen and breast tumor tissue were extracted with AccuZolTM reagent (Bioneer, Daedeok-gu, Daejeon, Korea) according to the manufacturer protocol. Subsequently, $1 \mu \mathrm{g}$ of total RNA was reverse transcribed into complementary DNA (cDNA) by MMLV reverse transcriptase and oligo-dT primer following manufacturer instructions (Promega, Madison, WI, USA). Relative gene expression was measured by real-time PCR using SYBR Premix Ex Taq (Takara Bio, Otsu, Shiga, Japan) and the Rotor-GeneTM 6000 system (Corbett Life Science, Mortlake, NSW, Australia). The real-time PCR was performed in a total $20 \mu \mathrm{l}$ reaction volume containing $1 \mu \mathrm{l}$ of cDNA template, $12 \mu \mathrm{l}$ of SYBR green reagent, $0.2 \mu \mathrm{M}$ of each of the primers and $6 \mu \mathrm{l}$ of nuclease-free distilled 
Dendrosomal Curcumin Suppresses Metastatic Breast Cancer in Mice by Changing M1/M2 Macrophage Balance

water. The sequences of PCR primers were as follows. The PCR conditions were setup $95^{\circ} \mathrm{C}$ for $10 \mathrm{~min}$ followed by 45 cycles at $95^{\circ} \mathrm{C}$ for $20 \mathrm{sec}$ and $60^{\circ} \mathrm{C}$ for $1 \mathrm{~min}$. The gene expression levels of IL-10, IL-12, STAT3, STAT4 and arginase normalized to $\beta$-actin were calculated using the relative quantification $(\Delta \Delta \mathrm{Ct})$ study software (Livak and Schmittgen, 2001), and the results are presented as arbitrary fold change compared to control.

\section{Statistical analysis}

Statistics were presented in Prism ${ }^{\circledR} 6.1$ software (GraphPad Software, Inc, La Jolla, CA, USA) and analyzed using one-way analysis of variance (ANOVA) followed by Newman-Keuls multiple comparison test or Student's t-test. All experiments were performed in triplicate. Differences among groups were stated to be statistically significant when $P<0.05$.

\section{Results}

\section{General observations}

Although Animals' weights were increased in both control and treated groups up to three weeks, they were not significantly different from each other. Body weight rose steadily to reach a plateau after about 3 weeks in all animals, but showed a slight fall in the control group in the last few weeks, which is expected due to tumor development. Except for a slight drop in food intake (g/day) in the control group, there were no significant difference in terms of food intake between control and treatment groups. No behavioral changes were observed in the animals during the course of administration, or in the follow up period.

\section{Tumor incidence and size}

At the end of the second week, tumor induction rate was $100 \%$ in control and treated groups. The tumor

A)

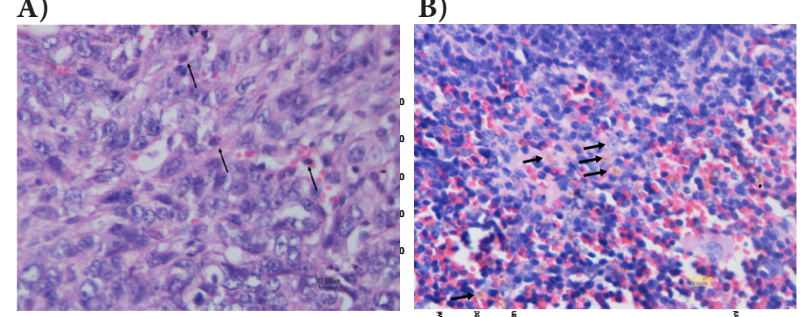

volume $\left(\mathrm{cm}^{3}\right)$ in mice treated with DNC was smaller in comparison with control. There were significant differences in the average tumor volume in different days between control and treatment groups. The mean final tumor volume reached approximately $1.11,0.31$ and $0.27 \mathrm{~cm}^{3}$ in the control, 40 and $80 \mathrm{mg} / \mathrm{kg}$ DNC groups, respectively (Figure 1). In addition, average final tumor weight reached approximately $1400 \mathrm{mg}$ in control and 550 and $500 \mathrm{mg}$ in 40 and $80 \mathrm{mg} / \mathrm{kg}$ DNC groups, respectively (Figure 2).
A)

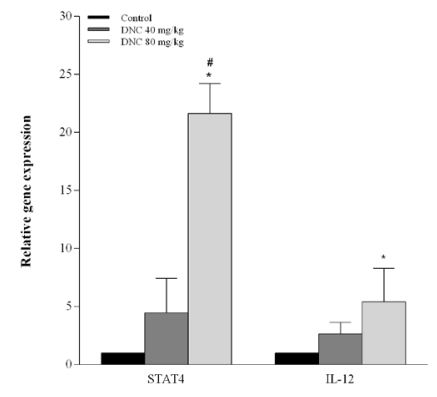

B)

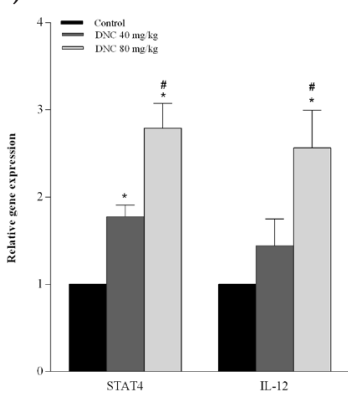

Figure 2. Effects of Dendrosomal Curcumin On Gene Expression of STAT4 and IL-12 in Tumor (2A) and Spleen (2B) Tissues. Data reported are mean \pm SD; ${ }^{~} P<0.05$ compared to $40 \mathrm{mg} / \mathrm{kg} \mathrm{DNC}$; $* P<0.05$ compared to control; $\mathrm{DNC}=$ dendrosomal curcumin

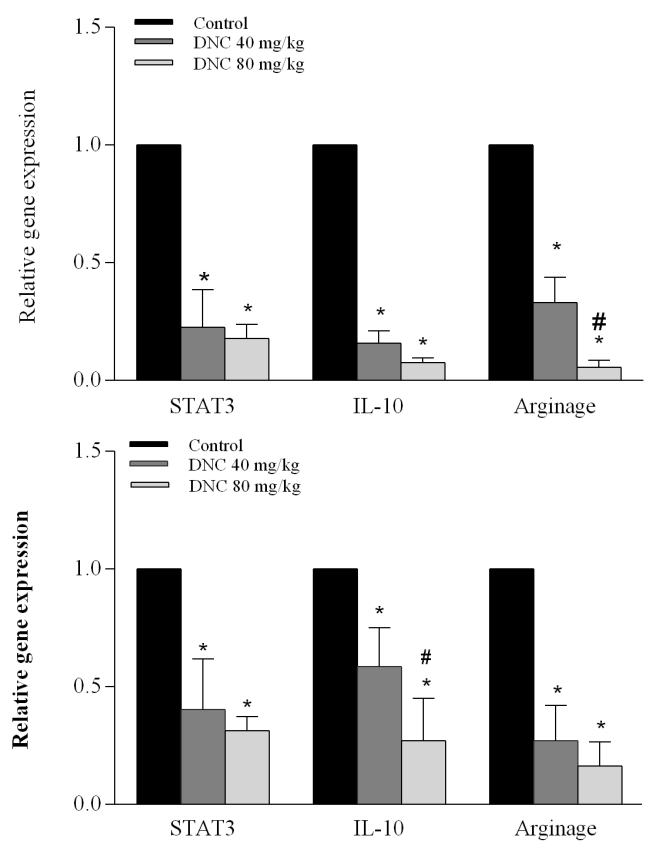

Figure 3. Effects of Dendrosomal Curcumin on Suppressing Gene Expression of STAT3, IL-10 and Arginase $I$ in Tumor (3A) and spleen (3B) tissues. Data reported are mean $\pm \mathrm{SD} ;{ }^{\#} P<0.05$ compared to $40 \mathrm{mg} / \mathrm{kg} \mathrm{DNC}$; $* P<0.05$ compared to control; $\mathrm{DNC}=$ dendrosomal curcumin

Table 1. Nucleotide Sequences of the Used Primers for Real-time RT-PCR

\begin{tabular}{lllc}
\hline Gene & Forward primer $\left(5^{\prime}-3^{\prime}\right)$ & Reverse primer $\left(5^{\prime}{ }^{\prime} 3^{\prime}\right)$ & Size $(\mathrm{bp})$ \\
\hline IL12 (p40) & GGAAGCACGGCAGCAGAATA & AACTTGAGGGAGAAGTAGGAATGG & 176 \\
STAT4 & CGCAGATAGTGAACGAGACAG & CCAGTTGCTGTCTGAGTTGG & 197 \\
IL-10 & CTATGCTGCCTGCTCTTACTGA3 & GGAGTCGGTTAGCAGTATGTTG & 200 \\
STAT3 & GTCGGTTGGAGGTGTGAGGTA & GGGAAAGGAAGGCAGGTTGA & 256 \\
Arginase I & GTCCACCCTGACCTATGTGTC & CAGGAGAAAGGACACAGGTTG & 176 \\
\hline
\end{tabular}


Sadaf Shiri et al

The presence of macrophages was confirmed in spleen and tumor tissues by $\mathrm{H} \& \mathrm{E}$ staining, indicated by arrows in (Figure 1).

\section{Analysis of gene expression by real-time PCR}

To confirm the presence of the different macrophages, using real-time PCR, we assessed IL-12 and STAT4 gene expression for characterization of M1, and IL-10, STAT3 and arginase I for characterization of M2 in spleen and tumor tissues. The results showed that IL-12 and STAT4 mRNA expression increased in 40 and $80 \mathrm{mg} / \mathrm{kg}$ DNC groups in comparison with controls. These increases were significant in the $80 \mathrm{mg} / \mathrm{kg}$ DNC -treated tumors (IL-12 mRNA; 5.41 fold and STAT4 mRNA; 21.65 fold) $(P<0.05)$ (Figure 2).

In a similar analysis for $\mathrm{M} 2$, the results indicated that IL-10 was significantly down-regulated in 40 and $80 \mathrm{mg} /$ $\mathrm{kg}$ of DNC-treated group compared to control in tumor and spleen tissues $(P<0.05)$. This reduction in $80 \mathrm{mg} / \mathrm{kg} \mathrm{DNC}$ was more pronounced in spleen tissue (spleen 0.27 fold) than $40 \mathrm{mg} / \mathrm{kg}$ DNC (spleen 0.5 fold) $(P<0.05)$. STAT3 mRNA expression was reduced in 40 and $80 \mathrm{mg} / \mathrm{kg}$ of DNC groups compared to control in tumor and spleen tissues $(P<0.05)$. Moreover, arginase I mRNA expression was decreased in 40 and $80 \mathrm{mg} / \mathrm{kg}$ DNC groups compared to control in tumor and spleen tissues $(P<0.05)$. This down-regulation was more significant in $80 \mathrm{mg} / \mathrm{kg}$ DNC (breast tumor 0.05 ) than $40 \mathrm{mg} / \mathrm{kg} \mathrm{DNC}$ group (breast tumor 0.33) $(P<0.05)$ (Figure 3).

\section{Discussion}

The present study examines the role of different macrophages on the effects of dendrosomal nanocurcumin in a typical animal model of metastatic breast cancer. Histopathological data showed the presence of macrophages in breast tumor and spleen tissues. After the course of treatment, tumor size and weight were significantly reduced in DNC-treated groups compared to control. Real-time PCR analyses demonstrated that DNC treatment increased STAT4 and IL-12 genes expression, which is indicative of the high level of M1 macrophage in tumor and spleen tissues. In addition, DNC decreased STAT3, IL-10 and arginase I genes expression, which in turn, refers to the lower number of M2 macrophage in tumor and spleen tissues. Overall, data indicates a increase in M1 subset and decrease in M2 macrophages. This change in $\mathrm{M} 1 / \mathrm{M} 2$ balance has a positive role in the protective effects of dendrosomal curcumin on mice metastatic breast cancer.

The results of our study demonstrated that DNC upregulates IL-12 and STAT4 in breast tumor and spleen tissues when used in different doses. STAT4 is a critical component of IL-12 signaling pathway (Huang et al., 2011), which causes up-regulation of its receptor. This action in turn, can facilitate the differentiation of M0 macrophage to M1 macrophage. This class of macrophages has a prominent role in inhibition of promotion and tumor proliferation. In this regard, a previous study has shown that curcumin differentiates myeloid-derived suppressor cells toward a M1 phenotype (Tu et al., 2012). This is well in line with the results of current study, showing that DNC inhibits the development of breast tumor, supposedly by increasing the ratio of M1 macrophages, and this can be regarded at least, as a partial anti-tumor mechanism for curcumin in breast cancer.

We also showed that DNC-in different doses, downregulates STAT3, IL-10 and arginase I in breast tumor and spleen tissues. STAT3 signaling pathway can promote the expression of IL-10 and arginase I in macrophages. Decreased STAT3 leads to reduction in IL-10 and arginase I expression (Yu et al., 2009; Vasquez-Dunddel et al., 2013), which can diminish the level of M2 macrophages at different tissues. M2 has a key role in tumor progression and growth (Hao et al., 2012; Medrek et al., 2012; Zhang et al., 2013b). Previous studies have demonstrated that M2 macrophages induce over-expression of STAT3 (Sica and Mantovani, 2012). There is evidence that STAT3 signaling pathway can trigger the cross-communication between tumor cells and M2 macrophages, that are crucial for the regulation of malignant progression (Zhang et al., 2013a), while abnormal STAT3 signaling has been associated with initiation and progression of several human cancers (Siveen et al., 2014). A number of other studies have also referred to the inhibitory effects of curcumin on M2 macrophages (Varalakshmi et al., 2008; Vishvakarma and Singh, 2010; Weiss et al., 2010; Srivastava et al., 2011). Furthermore, Curcumin directly inhibits IL-10 secretion, which can in turn inhibit JAK-STAT signaling and increase apoptosis (Alexandrow et al., 2012; Uddin et al., 2005; Yang et al., 2012). Zhang et al (2013), employed hydrazinocurcumin-encapsulated nanoparticles as an inhibitor of STAT3 activation, and through arginase I down-regulation, efficiently reactivated M0 macrophages towards M1 differentiation (Bounaama et al., 2012; Zhang et al., 2013a). It seems that curcumin can reduce M2 macrophage differentiation by down-regulation of STAT3, IL-10 and arginase I, and this finding can explain the inhibitory effects of DNC formulation on breast tumor in the present study.

It is important to note that the most important parameter for discriminating between M1 and M2 differentiation is the expression ratio of IL-12/IL-10 in tumor tissues (Heusinkveld and van der Burg, 2011; Wang et al., 2010). M1 macrophages typically express high level of IL-12 and low level of IL-10, and in comparison, M2 macrophages express high levels of IL-10 and low level of IL-12 (Heusinkveld and van der Burg, 2011; Wang et al., 2010). In the present study, IL-12 mRNA expression in tumor tissues increased in both $40 \mathrm{mg} / \mathrm{kg}$ (2.64 fold) and $80 \mathrm{mg} / \mathrm{kg}$ (5.4 fold) DNC-treated groups compared to control group. Furthermore, IL-10 mRNA expression decreased in $40 \mathrm{mg} / \mathrm{kg}$ ( 0.16 fold $)$ and $80 \mathrm{mg} / \mathrm{kg}$ ( 0.7 fold $)$ DNC-treated groups compared to control. According to the results of current study, M1 levels are seemingly affected more than M2 levels upon treatment with DNC.

In summary, our study indicates the protective effects of a dendrosomal curcumin formulation on mice metastatic breast cancer, witnessed by increase inrepresentation of M1 macrophages (confirmed by upregulation of STAT4 and IL-12), and decrease in M2 macrophages (confirmed by down-regulation of STAT3, 
Dendrosomal Curcumin Suppresses Metastatic Breast Cancer in Mice by Changing M1/M2 Macrophage Balance

IL-10 and arginase I). Future studies will clarify the characteristics, differentiation and number of M1 and M2 subpopulations by using immunohistochemistry and immunofluorescence techniques

\section{References}

Aggarwal BB, Sundaram C, Malani N, Ichikawa H (2007). Curcumin: the Indian solid gold. In The molecular targets and therapeutic uses of curcumin in health and disease (Springer), pp. 1-75.

Aggarwal BB, Sung B (2009). Pharmacological basis for the role of curcumin in chronic diseases: an age-old spice with modern targets. Trends Pharmacol Sci, 30, 85-94.

Alexandrow MG, Song LJ, Altiok S, et al (2012). Curcumin: a novel stat 3 pathway inhibitor for chemoprevention of lung cancer. Eur J Cancer Prev, 21, 407.

Alizadeh AM, Khaniki M, Azizian S, et al (2012). Chemoprevention of azoxymethane-initiated colon cancer in rat by using a novel polymeric nanocarrier-curcumin. European J Pharmacol, 689, 226-32.

Alizadeh AM, Shiri S, Farsinejad S (2014). Metastasis review: from bench to bedside. Tumor Biol, 35, 8483-523.

Antony S, Kuttan R, Kuttan G (1999). Immunomodulatory activity of curcumin. Immunol Invest, 28, 291-303.

Babaei E, Sadeghizadeh M, Hassan ZM, et al (2012). Dendrosomal curcumin significantly suppresses cancer cell proliferation $<\mathrm{i}>$ in vitro $</ \mathrm{i}>$ and $<\mathrm{i}>$ in vivo $</ \mathrm{i}>$. Int Immunopharmacol, 12, 226-34.

Bhattacharyya S, Hossain DMS, Mohanty S, et al (2010). Curcumin reverses $\mathrm{T}$ cell-mediated adaptive immune dysfunctions in tumor-bearing hosts. Cellular Mol Immunol, 7, 306-15.

Biswas SK, Chittezhath M, Shalova IN, Lim J-Y (2012). Macrophage polarization and plasticity in health and disease. Immunol Res, 53, 11-24.

Bounaama A, Djerdjouri B, Laroche-Clary A, Le Morvan V, Robert J (2012). Short curcumin treatment modulates oxidative stress, arginase activity, aberrant crypt foci, and TGF- $\beta 1$ and HES-1 transcripts in 1, 2-dimethylhydrazinecolon carcinogenesis in mice. Toxicol, 302, 308-17.

Colombo MP, Trinchieri G (2002). Interleukin-12 in anti-tumor immunity and immunotherapy. Cytokine Growth Factor Rev, 13, 155-68.

Cui Y-L, Li H-K, Zhou H-Y, Zhang T, Li Q (2013). Correlations of tumor-associated macrophage subtypes with liver metastases of colorectal cancer. Asian Pac J Cancer Prev, 14, 1003-7.

Ghalandarlaki N, Alizadeh AM, Ashkani-Esfahani S (2014). Nanotechnology-applied curcumin for different diseases therapy. Bio Med Res Int, 2014, 394264

Hao N-B, Lu M-H, Fan Y-H, et al (2012). Macrophages in tumor microenvironments and the progression of tumors. Clin Dev Immunol, 2012, 948098.

Heusinkveld M, van der Burg SH (2011). Identification and manipulation of tumor associated macrophages in human cancers. J Translat Med, 9, 216.

Huang Y, Lei Y, Zhang H, Zhang M, Dayton A (2011). Interleukin-12 treatment down-regulates STAT4 and induces apoptosis with increasing ROS production in human natural killer cells. J Leukocyte Biol, 90, 87-97.

Jagetia GC, Aggarwal BB (2007). "Spicing up" of the immune system by curcumin. J Clin Immunol, 27, 19-35.

Kamran MZ, Patil P, Gude RP (2013). Role of STAT3 in cancer metastasis and translational advances. Bio Med Res Int, 2013, 421821.

Kelloff GJ, Crowell JA, Steele VE, et al (2000). Progress in cancer chemoprevention: development of diet-derived chemopreventive agents. $J$ Nutr, 130, 467-71.

Khaniki M, Azizian S, Alizadeh AM, et al (2013). The antiproliferative and anticancerogenic effects of nanocurcumin in rat colon cancer. Tehran Univers Med J, 71, 277-284.

Livak KJ, Schmittgen TD (2001). Analysis of relative gene expression data using real-time quantitative PCR and the $2<$ sup $>-\Delta \Delta C \mathrm{C}</$ sup $>$ method. Methods, 25, 402-8.

Martinez FO, Sica A, Mantovani A, Locati M (2007). Macrophage activation and polarization. Frontiers in Bioscience: J Virtual Library, 13, 453-61.

Medrek C, Ponten F, Jirstrom K, Leandersson K (2012). The presence of tumor associated macrophages in tumor stroma as a prognostic marker for breast cancer patients. $B M C$ Cancer, 12, 306.

Mohsenikia M, Alizadeh AM, Khodayari S, et al (2013). The protective and therapeutic effects of alpha-solanine on mice breast cancer. Eur J Pharmacol, 718, 1-9.

Murray PJ, Wynn TA (2011). Protective and pathogenic functions of macrophage subsets. Nature Rev Immunol, 11, 723-37.

Niu G, Wright KL, Ma Y, et al (2005). Role of Stat 3 in regulating p53 expression and function. Mol Cell Bio, 25, 7432-40.

Olefsky JM, Glass CK (2010). Macrophages, inflammation, and insulin resistance. Ann Rev Phys, 72, 219-46.

Sadeghizadeh M, Ranjbar B, Damaghi M, et al (2008). Dendrosomes as novel gene porters-III. J Chem Technol Biotechnol, 83, 912-20.

Sarbolouki MN, Sadeghizadeh M, Yaghoobi MM, et al (2000). Dendrosomes: a novel family of vehicles for transfection and therapy. J Chem Technol Biotechnol, 75, 919-22.

Schindler H, Lutz MB, Rollinghoff M, Bogdan C (2001). The production of IFN- $\gamma$ by IL-12/IL-18-activated macrophages requires STAT4 signaling and is inhibited by IL-4. $J$ Immunol, 166, 3075-82.

Sica A, Mantovani A (2012). Macrophage plasticity and polarization: in vivo veritas. J Clin Invest, 122, 787-95.

Sica A, Schioppa T, Mantovani A, Allavena P (2006). Tumourassociated macrophages are a distinct M2 polarised population promoting tumour progression: potential targets of anti-cancer therapy. Eur J Cancer, 42, 717-27.

Sinha P, Clements VK, Bunt SK, Albelda SM, OstrandRosenberg S (2007). Cross-talk between myeloid-derived suppressor cells and macrophages subverts tumor immunity toward a type 2 response. J Immunol, 179, 977-83.

Siveen KS, Sikka S, Surana R, et al (2014). Targeting the STAT3 signaling pathway in cancer: Role of synthetic and natural inhibitors. Biochim Biophys Acta, 1845, 136-54.

Solinas G, Germano G, Mantovani A, Allavena P (2009). Tumorassociated macrophages (TAM) as major players of the cancer-related inflammation. J Leukocyte Biol, 86, 1065-73.

Srivastava RM, Singh S, Dubey SK, Misra K, Khar A (2011). Immunomodulatory and therapeutic activity of curcumin. Intern Immunopharmacol, 11, 331-41.

Tu SP, Jin H, Shi JD, et al (2012). Curcumin induces the differentiation of myeloid-derived suppressor cells and inhibits their interaction with cancer cells and related tumor growth. Cancer Prev Res, 5, 205-15.

Uddin S, Hussain AR, Manogaran PS, et al (2005). Curcumin suppresses growth and induces apoptosis in primary effusion lymphoma. Oncogene, 24, 7022-30.

Varalakshmi C, Ali AM, Pardhasaradhi B, et al (2008). Immunomodulatory effects of curcumin: $<\mathrm{i}>$ In-vivo $</ \mathrm{i}>$. Intern Immunopharmacol, 8, 688-700.

Vasquez-Dunddel D, Pan F, Zeng Q, et al (2013). STAT3 regulates arginase-I in myeloid-derived suppressor cells from cancer patients. J Clin Invest, 123, 1580-9. 
Sadaf Shiri et al

Vishvakarma NK, Singh SM (2010). Immunopotentiating effect of proton pump inhibitor pantoprazole in a lymphomabearing murine host: implication in antitumor activation of tumor-associated macrophages. Immunol Letters, 134, 83-92.

Wang W, Wang J, Dong S-f, et al (2010). Immunomodulatory activity of andrographolide on macrophage activation and specific antibody response. Acta Pharmacologica Sinica, 31, 191-201.

Weiss JM, Ridnour LA, Back T, et al (2010). Macrophagedependent nitric oxide expression regulates tumor cell detachment and metastasis after IL-2/anti-CD40 immunotherapy. J Exp Med, 207, 2455-67.

Xu M, Mizoguchi I, Morishima N, et al (2010). Regulation of antitumor immune responses by the IL-12 family cytokines, IL-12, IL-23, and IL-27. J Immunol Res, 2010, 832454.

Yang C-L, Liu Y-Y, Ma Y-G, et al (2012). Curcumin blocks small cell lung cancer cells migration, invasion, angiogenesis, cell cycle and neoplasia through Janus kinase-STAT3 signalling pathway. PLoS One, 7, 37960 .

Yu H, Jove R (2004). The STATs of cancer-new molecular targets come of age. Nature Rev Cancer, 4, 97-105.

Yu H, Pardoll D, Jove R (2009). STATs in cancer inflammation and immunity: a leading role for STAT3. Nature Rev Cancer, 9, 798-809.

Zhang X, Tian W, Cai X, et al (2013a). Hydrazinocurcumin encapsuled nanoparticles "re-educate" tumor-associated macrophages and exhibit anti-tumor effects on breast cancer following STAT3 suppression. PloS One, 8, 65896.

Zhang Y, Cheng S, Zhang M, et al (2013b). High-infiltration of tumor-associated macrophages predicts unfavorable clinical outcome for node-negative breast cancer. PloS One, 8, 76147. 